\title{
Uma Abordagem Baseada em Ontologias para Modelagem e Avaliação do Estudante em Sistemas Adaptativos e Inteligentes para Educação
}

\author{
Hiran N. M. Ferreira ${ }^{1,2}$, Rafael D. Araújo ${ }^{1}$, Fabiano A. Dorça ${ }^{1}$, Renan G. Cattelan ${ }^{1}$ \\ ${ }^{1}$ Faculdade de Computação - Universidade Federal de Uberlândia (UFU) \\ Uberlândia/MG, Brasil \\ ${ }^{2}$ Instituto Federal de Educação, Ciência e Tecnologia do Sul de Minas Gerais \\ (IFSULDEMINAS) - Passos/MG, Brasil
}

\begin{abstract}
New technologies emerge everyday with the aim of supporting the development of tools that are more adaptable to the particular needs of each user. One of the computational resources that strives to improve these processes are Adaptive and Intelligent Educational Systems (AIES)s, which possess the capacity to alter its characteristics in order to attend to the users' needs. This paper proposes an ontology-based approach for student modeling and assessment in AIESs. The main function of this model is to follow the activities developed by the student and to determine a level of performance based on their knowledge and behavior. Experiments with real students were developed, in which was possible to identify the main indicators to evaluate and monitor their progress during the use of a virtual environment.
\end{abstract}

Resumo. Novas tecnologias surgem diariamente com o intuito de auxiliar na criação de ferramentas cada vez mais adaptáveis às particularidades de cada usuário. Um dos recursos computacionais que busca melhorar esses processos são os Sistemas Adaptativos e Inteligentes para Educação (SAIE)s, que possuem a capacidade de alterar suas características para atender às necessidades individuais de cada estudante. Este artigo propõe uma abordagem baseada em ontologias para a modelagem e avaliação do estudante em SAIEs. A principal função desse modelo é acompanhar as atividades desenvolvidas pelo estudante e determinar um nível de desempenho com base no seu conhecimento e comportamento. Experimentos com estudantes reais foram desenvolvidos, no qual foi possível identificar os principais indicadores para avaliar e acompanhar o seu progresso durante a utilização de um ambiente virtual.

\section{Introdução}

Nos últimos anos um grande número de sistemas adaptativos tem surgido com o intuito de auxiliar e apoiar usuários na realização de suas atividades. Esses sistemas, em sua maioria, baseiam-se em técnicas inteligentes para se adaptar às reais necessidades de cada usuário. Um dos cenários que vêm ganhando destaque para a aplicação desses conceitos são os ambientes educacionais. Quando aplicado de forma efetiva, esses ambientes educacionais que recebem características adaptativas tornam-se mais adequados para auxiliar o processo de ensino/aprendizagem [Brusilovsky 2001]. 
No contexto educacional, uma das características que impactam diretamente na eficácia da adaptabilidade é a identificação do nível de conhecimento do estudante. Conseguir identificar de forma efetiva o seu real estado cognitivo é uma das etapas mais importantes no processo de personalização. Algumas técnicas computacionais têm surgido com o intuito de auxiliar neste processo. Uma das mais importantes é a Learning Analytics, definida como a medição, coleta e análise de dados sobre o comportamento dos estudantes em ambientes educacionais com o objetivo de realizar uma interpretação individualizada do percurso, das dificuldades e das características de estudos dos estudantes [Siemens e Baker 2012].

Ainda é pouco explorado na literatura abordagens que integram ontologias ao processo de análise comportamental do estudante por meio de técnicas de Learning Analytics. Além de permitir reasoning por meio das informações representadas, as ontologias são capazes de fornecer estrutura conceitual comum para o desenvolvimento de bases de conhecimento compartilháveis e reutilizáveis. Além do mais, as ontologias facilitam a interoperabilidade e a fusão das informações que viabilizam a criação de aplicações computacionais poderosas e mais inteligentes [Isotani e Bittencourt 2015].

Neste sentido, este trabalho apresenta uma abordagem baseada em ontologias capaz de acompanhar atividades comportamentais dos estudantes e inferir sobre o seu real nível de desempenho, possibilitando melhor adaptação e personalização de conteúdo educacional com as reais necessidades de cada estudante. A representação por meio de ontologias foi escolhida por sua capacidade para a representação de conceitos e propriedades, pela sua característica que permite reuso e extensão, bem como, pela possibilidade de realizar inferências a partir das informações representadas.

O restante deste artigo está estruturado da seguinte maneira: na Seção 2, é apresentado um levantamento sobre o estado da arte acerca da utilização de ontologias no contexto educacional; na Seção 3, são descritos os detalhes da abordagem proposta; na Seção 4, é apresentada uma arquitetura de integração do modelo proposto a um SAIE; na Seção 5, são discutidos experimentos e resultados do trabalho; e, por fim, na Seção 6, são apresentadas as considerações finais e potenciais trabalhos futuros.

\section{Estado da Arte: Por que ontologia?}

Segundo Gruber [Gruber 1993], uma ontologia pode ser definida como "uma especificação explícita de uma conceitualização", ou seja, uma formalização dos conceitos e relacionamentos em um domínio específico. Na prática, uma ontologia define uma "linguagem", conjunto de termos, que será utilizada para formular consultas [Almeida e Bax 2003].

Uma ontologia descreve as regras de combinação entre os termos e seus relacionamentos para que usuários elaborem consultas usando os conceitos especificados. Segundo Yu [Yu 2011], os principais benefícios na utilização das ontologias são: (i) permitir interpretação e compartilhamento sobre conceitos de um domínio específico; (ii) permitir reutilização do domínio de conhecimento; (iii) permitir descrever o conhecimento e a semântica para que computadores possam compreender o domínio descrito. Mesmo com essas características, Gruber [Gruber 1993] assegura que o principal propósito da construção de ontologias é possibilitar o compartilhamento e a reutilização de conhecimento. 
VI Congresso Brasileiro de Informática na Educação (CBIE 2017)

Anais do XXVIII Simpósio Brasileiro de Informática na Educação (SBIE 2017)

O uso de ontologias para representar modelos de usuários tem atraído, cada dia mais, a atenção de pesquisadores. Chrysafiadi e Virvou [Chrysafiadi e Virvou 2013] mostram um crescente número de trabalhos que abordam essa técnica para modelagem de informações educacionais. SoNITS [Nguyen et al. 2011] é um Modelo do Estudante (ME) fundamentado em ontologias para um ambiente educacional baseado em redes sociais com o intuito de ajudar na organização do conhecimento e do raciocínio sobre as relações de habilidade. Clemente e colaboradores [Clemente et al. 2011] apresentam o MAEVIF, uma plataforma voltada ao desenvolvimento de ambientes educacionais para treinamentos. Esse ambiente proporciona conteúdo personalizado e fornece o feedback mais adequado em cada instante da interação por meio de um ME, que é baseado em ontologias e regras de diagnóstico. No trabalho de Panagiotopoulos [Panagiotopoulos et al. 2012] é proposto um ME baseado em ontologias para ambiente educacional voltado à educação a distância. A ontologia é capaz de representar localização, domínio de conhecimento, objetivos, estilos de aprendizado e afeto.

No trabalho de Rezende e colaboradores [Rezende et al. 2015] é descrita uma ontologia para a modelagem do perfil e do contexto do estudante em um ambiente $e$ learning, a qual visa padronizar as informações e facilitar o processo de recomendação de objetos de aprendizagem. No trabalho apresentado por [Nafea et al. 2016] é proposto um novo modelo para definição automática do perfil de estudante. A abordagem baseia-se em padrões reais de comportamento dos estudantes durante a interação com um portal educacional e emprega ontologias e mecanismos de inferências para identificar estilos de aprendizagem.

Pukkhem [Pukkhem 2013] propõe um framework baseado em ontologias e regras de mapeamento semântico para personalização baseada no estilo de aprendizagem do estudante. Este framework emprega ontologias para que computadores sejam capazes de interpretar e processar objetos de aprendizagem em um sistema de recomendação. Ouf e colaboradores [Ouf et al. 2017] propõem e implementam um framework para smart elearning que utiliza ontologias e regras SWRL (Semantic Web Rule Language). Uma ontologia é utilizada para representar um modelo de aprendizagem e outras três para representar todos os componentes do processo de aprendizagem (objetos de aprendizagem, atividades de aprendizagem e métodos de ensino). Em ambos os trabalhos, regras SWRL são utilizadas para auxiliar no processo de inferências sobre características do usuário, não sobre questões comportamentais e de desempenho.

Como pode ser observado, a utilização de ontologias para representação das características dos estudantes é aceita e muito empregada pela comunidade acadêmica. Tal fato, se deve, principalmente, pelas características de representação de domínio, possibilidade de permitir extensão e reutilização, e por sua capacidade de processar novos conhecimentos a partir de inferências realizadas através das informações representadas na ontologia. Outro detalhe que pode ser observado é que, raramente, as ontologias estão sendo empregadas para auxiliar no processo de monitoramento e análise comportamental do estudante, apresentado assim, uma lacuna nas pesquisas relacionadas com Learning Analitics. Diferentemente do trabalho aqui proposto, nenhum dos trabalhos citados explora essa capacidade. 
VI Congresso Brasileiro de Informática na Educação (CBIE 2017)

Anais do XXVIII Simpósio Brasileiro de Informática na Educação (SBIE 2017)

\section{Abordagem Proposta}

Com o intuito de levantar os principais dados a serem representados na ontologia, foi conduzido um estudo com o objetivo de levantar as principais características necessárias para a descrição de um estudante, para, assim, criar um modelo que fosse conciso e que representasse as principais informações para esses ambientes. Essas informações devem ser capazes de modelar, de forma virtual, todas as características dos estudantes que influenciam para a descrição do comportamento e das atividades desenvolvidas no ambiente que possam influenciar na tomada de decisão para adaptação e personalização de conteúdo. As características representadas neste modelo estão classificadas em quatro dimensões: Informações Pessoais, Contexto, Comportamento e Conhecimento. Essas dimensões foram propostas com base em diversos modelos anteriormente criados e que tiveram repercussões positivas na comunidade acadêmica [Ting e Phon-Amnuaisuk 2012, Shute et al. 2015].

$\mathrm{Na}$ literatura é possível encontrar diversas metodologias que orientam o processo de construção de ontologias. De acordo com BautistaZambrana [Bautista-Zambrana 2015], esses métodos envolvem um conjunto de atividades, como conceitualização, formalização, implementação e manutenção. Para a construção da ontologia proposta, foi utilizado o método $101^{1}$. Além de considerar as principais fases na modelagem de uma ontologia, é capaz de utilizar ferramentas especializadas para auxiliar no processo de desenvolvimento. Os principais processos representados por essa metodologia são: (i) determinar o domínio e o escopo da ontologia; (ii) considerar a reutilização de ontologias existentes; (iii) enumerar termos importantes na ontologia; (iv) definir classes e hierarquia de classes; (v) definir as propriedades de classes/slots; (vi) definir as restrições; (vii) criar as instâncias.

Seguindo a abordagem proposta pelo método, foi criada uma ontologia que apresenta características suficientes para abstração de um modelo que seja capaz de representar o estado atual de conhecimento dos estudantes, possibilitando melhor adaptação e personalização de conteúdo educacional. Um detalhe interessante da abordagem proposta neste trabalho é que o domínio de conhecimento não está integrado à ontologia e sim a um outro modelo probabilístico, garantindo que a ontologia possa ser empregada em outros domínios sem a necessidade de alterações.

A Tabela 1 apresenta as principais classes definidas para a ontologia. É possível observar também na tabela algumas referências encontradas na literatura. Para a definição da hierarquia de classes, seguiu-se o modelo proposto por Uschold e Gruninger [Uschold e Gruninger 1996], que faz uma combinação entre a abordagem Top-Down e Bottom-Up.

\subsection{Base de Regras}

Uma das grandes vantagens de utilizar ontologias está justamente na sua capacidade de processar inferências. Para processar essas inferências, regras SWRL foram criadas, a fim de proporcionar um resultado mais condizente com as reais capacidades dos estudantes, pois além de analisarem o conhecimento, também consideram aspectos comportamentais (nível de colaboração, nível de gamificação, atividades desenvolvidas, etc.).

\footnotetext{
${ }^{1}$ referenciada no Inglês como Ontology Development 101
} 
VI Congresso Brasileiro de Informática na Educação (CBIE 2017)

Anais do XXVIII Simpósio Brasileiro de Informática na Educação (SBIE 2017)

Tabela 1. Principais classes representadas na ontologia.

\begin{tabular}{|c|c|c|c|}
\hline $\mathrm{N}^{1}$ & Classe & Descrição & Referência \\
\hline$i$ & Student & Representa o estudante no ambiente educacional & $\begin{array}{l}\text { [Mahnane e Laskri 2012, } \\
\text { Rezende et al. 2015] }\end{array}$ \\
\hline$i i$ & $\begin{array}{l}\text { Personal Informa- } \\
\text { tion }\end{array}$ & Informações como nome, email, idade, sexo, etc. & $\begin{array}{l}\text { [Dolog e Nejdl 2007, } \\
\text { Rezende et al. 2015] }\end{array}$ \\
\hline$i i$ & Learning Style & $\begin{array}{l}\text { Estilo de Aprendizagem baseado no modelo de Fel- } \\
\text { der e Silverman }\end{array}$ & [Mahnane e Laskri 2012] \\
\hline$i i$ & Preferences & $\begin{array}{l}\text { Classe genérica para representar a preferência de } \\
\text { um estudante }\end{array}$ & $\begin{array}{l}\text { [Dolog e Nejdl 2007, } \\
\text { Rezende et al. 2015] }\end{array}$ \\
\hline$i i i$ & Context & Representação do contexto de acesso & [Rezende et al. 2015] \\
\hline$i i i$ & Device & Dispositivo usado pelo estudante & $\begin{array}{l}\text { [Verbert et al. 2012, } \\
\text { Rezende et al. 2015] }\end{array}$ \\
\hline$i i i$ & Location & Localização do estudante durante o acesso & $\begin{array}{l}\text { [Verbert et al. 2012, } \\
\text { Rezende et al. 2015] }\end{array}$ \\
\hline$i v$ & Knowledge State & Estado de conhecimento do estudante & [Nguyen et al. 2011] \\
\hline iv & Knowledge & Representa o conhecimento em um conceito & $\begin{array}{l}\text { [Dolog e Nejdl 2007, } \\
\text { Clemente et al. 2011] }\end{array}$ \\
\hline$i v$ & Course & Unidade educacional & [Verbert et al. 2012] \\
\hline$i v$ & Content & Conteúdo de um curso & [Verbert et al. 2012] \\
\hline$v$ & Behavior & $\begin{array}{l}\text { Comportamento do estudante durante a utilização } \\
\text { do ambiente educacional }\end{array}$ & [Clemente et al. 2011] \\
\hline$v$ & Collaboration & Atv. de colaboração: comentários, ratings, etc. & [Hsiao et al. 2013] \\
\hline$v$ & Gamification & Pontuação em atividades de gamificação & $\begin{array}{l}\text { [Shute et al. 2015, } \\
\text { Minović et al. 2015] }\end{array}$ \\
\hline$v$ & Game & Mecanismo de game disponível para pontuação & $\begin{array}{l}\text { [Shute et al. 2015, } \\
\text { Minović et al. 2015] }\end{array}$ \\
\hline$v i$ & Trace & Rastro de atividades executadas no acesso & [Clemente et al. 2011] \\
\hline$v i$ & Activity & Atividade executada durante um acesso & [Shute et al. 2015] \\
\hline
\end{tabular}

${ }^{1}$ Decomposição Modular [Suárez-Figueroa et al. 2008].

As regras propostas para esse modelo baseiam-se nas informações representadas pela ontologia combinadas com os valores de inferência já realizados por um modelo probabilístico [Ferreira et al. 2016] e produzem um resultado que indica o nível de desempenho do estudante. Um detalhe interessante deste modelo é justamente a capacidade de extensão e inclusão de novas regras, visto que cada domínio traz suas próprias características e particularidades.

Para este modelo foram propostas quatro categorias de regras: (i) Desempenho do estudante a partir das atividades de Socialização/Colaboração; (ii) Desempenho do estudante a partir das atividades de Gamificação; (iii) Desempenho do estudante a partir de instrumentos avaliativos, e por fim, (iv) Desempenho do estudante a partir das atividades desempenhadas durante a utilização do ambiente. Para cada categoria, foram criadas quatro subcategorias mais específicas, que podem agrupar uma ou mais regras.

A Tabela 2 apresenta algumas regras, em SWRL, que estão disponíveis no modelo. Vale ressaltar que esses são somente alguns exemplos de utilização de regras na ontologia, outras podem ser facilmente criadas. 
VI Congresso Brasileiro de Informática na Educação (CBIE 2017)

Anais do XXVIII Simpósio Brasileiro de Informática na Educação (SBIE 2017)

Tabela 2. Algumas Regras disponíveis na Base de Regras.

\begin{tabular}{|c|c|c|}
\hline & Regra $S W R L$ & Métricas \\
\hline 1 & $\begin{array}{l}\text { grade }(? \mathrm{~K}, \mathrm{~B}) \wedge \text { hasknowledge }(? \mathrm{~S}, ? \mathrm{~K}) \wedge \text { hascontent }(? \mathrm{~K}, ? \mathrm{C}) \wedge \\
\text { hasGamification (?S, ?G) } \wedge \text { gamVal }(? \mathrm{G}, \mathrm{high}) \rightarrow \text { hasLevel (?S, A) }\end{array}$ & $\begin{array}{l}\text { Conhecimento, } \\
\text { Gamificação }\end{array}$ \\
\hline 2 & 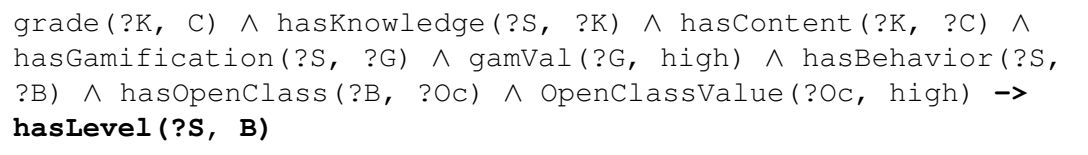 & $\begin{array}{l}\text { Conhecimento, } \\
\text { Gamificação, Compor- } \\
\text { tamento }\end{array}$ \\
\hline 3 & $\begin{array}{l}\text { grade }(? \mathrm{~K}, \mathrm{D}) \wedge \text { hasknowledge }(? \mathrm{~S}, \mathrm{?K}) \wedge \text { hascontent }(? \mathrm{~K}, \\
\text { ?C) } \wedge \text { hasGamification (?S, ?G) } \wedge \text { gamVal (?G, low) } \wedge \\
\text { hasBehavior(?S, ?B) } \wedge \text { hasVisualizationClass (?B, ?VC) } \wedge \\
\text { VisualizationClassValue(?VC, low) } \rightarrow \text { hasLevel(?S, D) }\end{array}$ & $\begin{array}{l}\text { Conhecimento, } \\
\text { Gamificação, Compor- } \\
\text { tamento }\end{array}$ \\
\hline 4 & $\begin{array}{l}\text { grade (?K, B) } \wedge \text { hasknowledge(?S, ?K) } \wedge \text { hascontent }(? K, ? C) \wedge \\
\text { hasBehavior(?S, ?B1) } \wedge \text { hasVisualizationclass(?B1, ?VC) } \wedge \\
\text { VisualizationClassValue (?VC, high) } \wedge \text { hasBehavior(?S, ?B2) } \\
\wedge \text { hasopenclass(?B2, ?OC) } \wedge \text { OpenClassValue(?OC, high) } \rightarrow> \\
\text { hasLevel(?S, A) }\end{array}$ & $\begin{array}{l}\text { Conhecimento, Com- } \\
\text { portamento }\end{array}$ \\
\hline
\end{tabular}

\section{Integração da Abordagem a um Ambiente Adaptativo}

Em um recente artigo, Luckin e Holmes [Luckin e Holmes 2016] discutem sobre a evolução na integração de recursos de Inteligencia Artificial com o intuito de acrescentar melhorias aos ambientes educacionais. Uma das discussões proposta no artigo é justamente a estruturação de modelos para apoiarem a adaptação de conteúdo individualizado em sistemas adaptativos para educação. Em uma abordagem geral, ele apresenta a estruturação e uma descrição dos principais componentes de um SAIE.

A Figura 1 apresenta uma adaptação da estrutura proposta por Luckin e Holmes, relacionando os principais módulos de um SAIE e como a ontologia proposta se comunica com eles. Conforme já proposto, a estrutura de um sistema adaptativo deve possuir três modelos (domínio, pedagógico e estudante) e um outro modelo capaz de realizar a apresentação do conteúdo ao estudante.

A abordagem proposta neste artigo insere uma ontologia para comunicação entre todos os modelos, sendo capaz, além de descrever as características do estudante, realizar o processamento do conhecimento através de inferências por meio de uma base de regras SWRL. A ontologia proposta recebe informações dos três modelos e gera um nível de desempenho, que será repassado ao módulo de interface para apresentação.

É possível notar na Figura 1 a presença da ontologia proposta conectada diretamente aos três principais modelos presentes no ambiente (pedagógico, domínio e estudante). Junto à ontologia é possível verificar uma base SWRL, que é utilizada para armazenar as regras para inferência sobre o nível de desempenho do estudante. Por fim, é possível ver que existe uma comunicação entre a ontologia e a interface, esta por sua vez, possui dois módulos, o primeiro responsável por adaptar o conteúdo ao contexto do usuário e o segundo responsável por acompanhar as interações dos usuários com o sistema. A interface comunica diretamente com o Modelo Aberto do Estudante trocando informações sobre o nível de desempenho dos estudantes. 
VI Congresso Brasileiro de Informática na Educação (CBIE 2017)

Anais do XXVIII Simpósio Brasileiro de Informática na Educação (SBIE 2017) Figura 1. Estrutura de Integração do Modelo Ontológico a um Sistema Adapta-
tivo. Adaptada de Luckin e Holmes [Luckin e Holmes 2016]

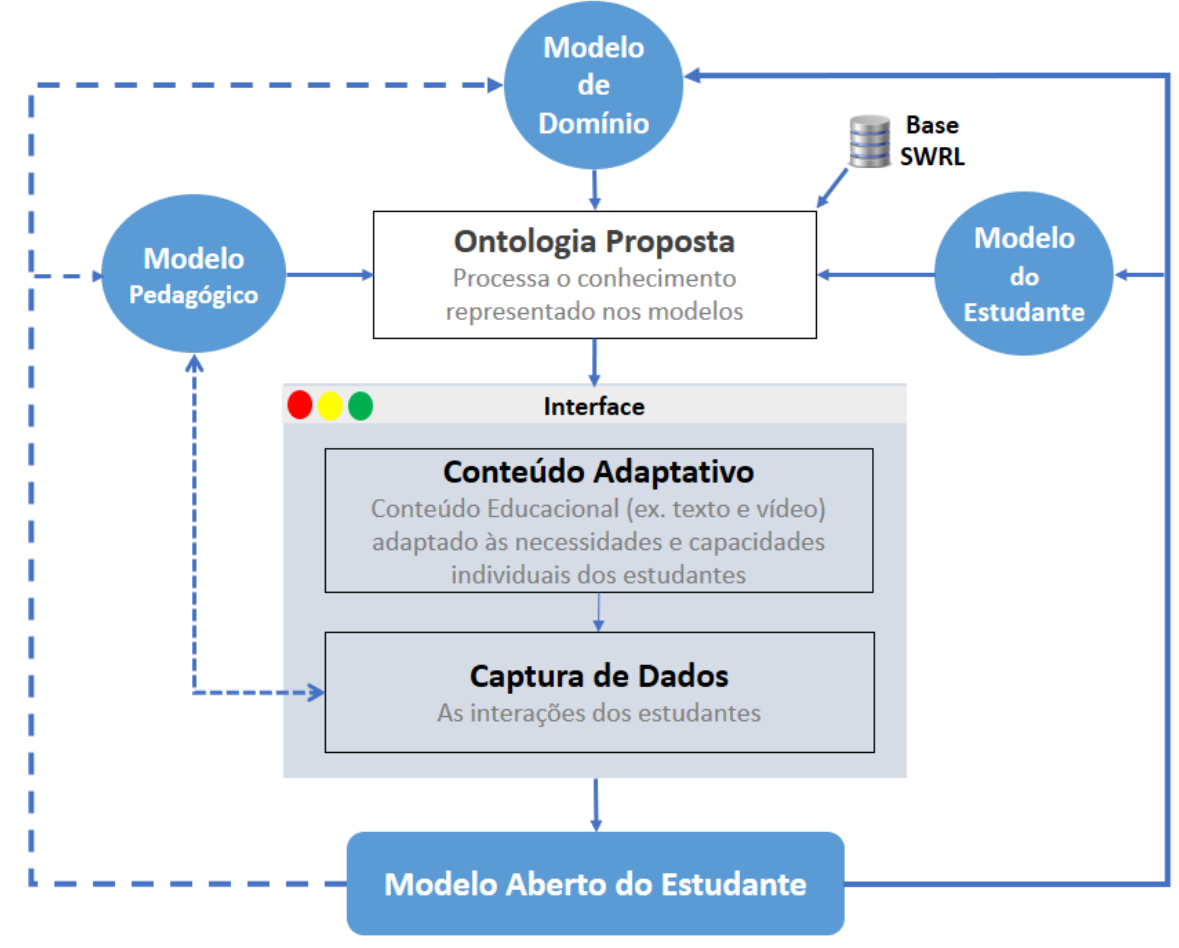

\section{Experimentos e Resultados}

A fim de avaliar as inferências realizadas pela ontologia, foram conduzidos experimentos com duas turmas de graduação na disciplina de Interação Humano-Computador no curso de Sistemas de Informação da Universidade Federal de Uberlândia (UFU). O modelo proposto foi integrado a um ambiente virtual de aprendizagem, o qual foi utilizado como ferramenta de apoio para as disciplinas presenciais. A primeira disciplina foi ministrada no primeiro semestre de 2016 e a segunda no segundo semestre de 2016. No total, 73 estudantes foram considerados neste experimento.

O principal objetivo da análise foi verificar se os conceitos inferidos pelas regras ontológicas correspondem corretamente às notas obtidas por meio de avaliações presenciais aplicadas pelo professor. É interessante notar que uma boa relação entre esses valores não significa que os valores inferidos sejam iguais aos reais, mas que esses valores (inferidos) correspondam aos esperados, levando em consideração o comportamento dos estudantes.

A Tabela 3 apresenta dados sobre a relação entre os valores inferidos pelo modelo através do sistema e os valores obtidos pelo professor através de instrumentos tradicionais. Inicialmente é possível notar que na Turma 2 houve um maior índice de resultados equivalentes entre os conceitos que na Turma 1 (índice $i$ na tabela); e também na Turma 2 houve maior influência positiva sobre a nota inferida pelo modelo, ou seja, o comportamento do estudante influenciou positivamente neste caso (índice $i i$ na tabela). Esse resultado pode ser comprovado analisando o comportamento dos estudantes na utilização do ambiente, na Turma 2 houve quase duas vezes mais interações que na Turma 1 (na Turma 2 houve 36.532 interações, ao passo que na Turma 1 houve 20.260 interações). 
Tabela 3. Relação entre os conceitos obtidos pelo professor e os conceitos inferidos pelo modelo.

\begin{tabular}{cccc}
\hline & Influência & Turma 1 (\%) & Turma 2 (\%) \\
\hline$i$ & $\odot^{1}$ & 21,2 & 52,0 \\
$i i$ & $\triangle^{2}$ & 15,1 & 37,0 \\
$i i i$ & $\nabla^{3}$ & 45,4 & 10,0 \\
$i v$ & $\triangle^{4}$ & 0,0 & 0,0 \\
$v$ & $\nabla \nabla^{5}$ & 18,1 & 0,0
\end{tabular}

${ }^{1}$ Conceitos Equivalentes ${ }^{2}$ Aumento do conceito inferido em relação ao real. ${ }^{3}$ Diminuição do conceito inferido em relação ao real. ${ }^{4}$ Aumento em dois ou mais níveis do conceito inferido em relação ao real. ${ }^{5}$ Diminuição em dois ou mais níveis do conceito inferido em relação ao real.

Como a quantidade de interações ocorridas na Turma 1 foi menor que na Turma 2, é possível comprovar os resultados indicados pelos índices iii e $v$ na Tabela 3. É possível notar que na Turma 1 o comportamento do estudante influenciou mais negativamente no seu conceito, indicando que, quando o nível de comportamento é menor, o modelo pode indicar um conceito menos satisfatório ao estudante. Por fim, é possível notar que em nenhuma turma houve a ocorrência do aumento de mais de dois níveis no valor inferido (como indica o índice $i v$ ). Isso indica que entre as regras criadas não foi possível inferir tal situação.

Outra análise que foi realizada teve como propósito verificar quais métricas tiveram maiores influências nos resultados. Foi possível observar que as métricas relacionadas com a interação no material disponibilizado pelo professor (slides das aulas) influenciaram no conceito dos estudantes, ou seja, estudantes que obtiveram uma diminuição nos conceitos (índice iii ou $v$ na tabela 3 ) foram justamente os estudantes que menos interagiram com o material educacional. Foi possível observar também que tanto as interações no material educacional quanto as atividades de gamificação contribuíram para a elevação do conceito inferido (índice $i i$ e $i v$ na tabela 3 ). Foi possível verificar que, sempre que o estudante conseguiu um alto valor nas atividades de gamificação, o seu nível de desempenho foi melhorado.

\section{Considerações Finais}

Este artigo apresentou uma abordagem baseada em ontologias para modelagem e avaliação de estudante. A modelagem proposta é concisa e, baseando-se em modelos disponíveis na literatura, agrupa os detalhes mais importantes para representação de um estudante em um sistema educacional. Quando aplicada a ambientes presenciais, essa abordagem consegue auxiliar no processo de avaliação, pois além de considerar o conhecimento, também considera o comportamento dos estudantes. Dassa forma, utilizando este modelo, professores podem obter avaliações mais refinadas, pois irão considerar diversos aspectos do comportamento de cada estudante.

Para validação da proposta foram realizados experimentos com estudantes reais, nos quais buscou-se avaliar a relação entre os valores inferidos pelo modelo e os valores obtidos pelos professores por meio de instrumentos tradicionais e, a partir disso, verificar quais fatores influenciaram positiva e negativamente no conceito final determinado pelo 
VI Congresso Brasileiro de Informática na Educação (CBIE 2017)

Anais do XXVIII Simpósio Brasileiro de Informática na Educação (SBIE 2017)

modelo. A partir das regras disponibilizadas no modelo, foi possível verificar que as atividades de abertura, visualização de slides e gamificação contribuíram, de alguma forma, para o refinamento do conceito final.

Potenciais trabalhos futuros incluem: a construção de regras semânticas para diagnóstico das principais dificuldades dos estudantes; a implementação de mecanismos para auxiliar na recomendação individualizada de conteúdo; e a realização de experimentos com mais estudantes.

\section{Agradecimentos}

Os autores agradecem o apoio das agências de pesquisa e instituições CNPq, CAPES, FAPEMIG, PROPP/UFU, FACOM/PPGCO/PROAP/UFU, IFSULDEMINAS e PET/MEC/SESu.

\section{Referências}

Almeida, M. B. e Bax, M. P. (2003). Uma visão geral sobre ontologias: Pesquisa sobre definições, tipos, aplicações, métodos de avaliação e de construção. Ciência da Informação, Brasília, 32(3):7-20.

Bautista-Zambrana, M. R. (2015). Methodologies to Build Ontologies for Terminological Purposes. Procedia - Social and Behavioral Sciences, 173:264-269.

Brusilovsky, P. (2001). Adaptive hypermedia. User Modeling and User-Adapted Interaction, 11(1-2):87-110.

Chrysafiadi, K. e Virvou, M. (2013). Student modeling approaches: A literature review for the last decade. Expert Systems with Applications, 40(11):4715-4729.

Clemente, J., Ramírez, J., e de Antonio, A. (2011). A proposal for student modeling based on ontologies and diagnosis rules. Expert Systems with Applications, 38(7):8066-8078.

Dolog, P. e Nejdl, W. (2007). The adaptive web. chapter Semantic Web Technologies for the Adaptive Web, pages 697-719. Springer, Berlin, Heidelberg.

Ferreira, H. N. M., Brant-Ribeiro, T., Araujo, R. D., Dorça, F. A., e Cattelan, R. G. (2016). An automatic and dynamic student modeling approach for adaptive and intelligent educational systems using ontologies and bayesian networks. In Proceedings of the 28th IEEE International Conference on Tools with Artificial Intelligence, ICTAI 2016, pages $738-745$.

Gruber, T. R. (1993). A translation approach to portable ontology specifications. Knowledge acquisition, 5(2):199-220.

Hsiao, I.-H., Bakalov, F., Brusilovsky, P., e König-Ries, B. (2013). Progressor: social navigation support through open social student modeling. New Review of Hypermedia and Multimedia, 19(2):112-131.

Isotani, S. e Bittencourt, I. I. (2015). Dados Abertos Conectados: Em busca da Web do Conhecimento. Novatec Editora.

Luckin, R. e Holmes, W. (2016). Intelligence unleashed: An argument for ai in education. 
VI Congresso Brasileiro de Informática na Educação (CBIE 2017)

Anais do XXVIII Simpósio Brasileiro de Informática na Educação (SBIE 2017)

Mahnane, L. e Laskri, M. T. (2012). An adaptive hypermedia system integrating thinking style (ahs-ts): Model and experiment. International Journal of Hybrid Information Technology, 5(1):11-28.

Minović, M., Milovanović, M., Šošević, U., e González, M. Á. C. (2015). Visualisation of student learning model in serious games. Computers in Human Behavior, 47:98-107.

Nafea, S., Maglaras, L., Siewe, F., Smith, R., e Janicke, H. (2016). Personalized students' profile based on ontology and rule-based reasoning. ICST Transactions 3:1-14,

Nguyen, C. D., Vo, K. D., Bui, D. B., e Nguyen, D. T. (2011). An ontology-based it student model in an educational social network. In Proceedings of the 13th International Conference on Information Integration and Web-based Applications and Services, iiWAS '11, pages 379-382, ACM.

Ouf, S., Ellatif, M. A., Salama, S., e Helmy, Y. (2017). A proposed paradigm for smart learning environment based on semantic web. Computers in Human Behavior, 72:796818.

Panagiotopoulos, I., Kalou, A., Pierrakeas, C., e Kameas, A. (2012). An ontology-based model for student representation in intelligent tutoring systems for distance learning. Artificial Intelligence Applications and Innovations, pages 296-305.

Pukkhem, N. (2013). Ontology-based semantic approach for learning object recommendation. International Journal on Information Technology, 3(4):12.

Rezende, P. A., Pereira, C., Campos, F., David, J., e Braga, R. (2015). Personna: proposta de ontologia de contexto e perfil de alunos para recomendação de objetos de aprendizagem. Revista Brasileira de Informática na Educação, 23(01):70.

Shute, V. J., D’Mello, S., Baker, R., Cho, K., Bosch, N., Ocumpaugh, J., Ventura, M., e Almeda, V. (2015). Modeling how incoming knowledge, persistence, affective states, and in-game progress influence student learning from an educational game. Computers \& Education, 86:224 - 235 .

Siemens, G. e Baker, R. S. J. d. (2012). Learning analytics and educational data mining: Towards communication and collaboration. In Proceedings of the $2 \mathrm{Nd}$ International Conference on Learning Analytics and Knowledge, LAK '12, pages 252-254, ACM.

Suárez-Figueroa, M. C., de Cea, G. A., Buil, C., Dellschaft, K., Fernández-López, M., García, A., Gómez-Pérez, A., Herrero, G., Montiel-Ponsoda, E., Sabou, M., et al. (2008). D5. 4.1. neon methodology for building contextualized ontology networks. NeOn Project Deliverable D, 5.

Ting, C.-Y. e Phon-Amnuaisuk, S. (2012). Properties of bayesian student model for inqpro. Applied Intelligence, 36(2):391-406.

Uschold, M. e Gruninger, M. (1996). Ontologies: Principles, methods and applications. Knowledge Engineering Review, 11:93-136.

Verbert, K., Manouselis, N., Ochoa, X., Wolpers, M., Drachsler, H., Bosnic, I., e Duval, E. (2012). Context-aware recommender systems for learning: A survey and future challenges. IEEE Transactions on Learning Technologies, 5(4):318-335.

Yu, L. (2011). A developer's guide to the semantic Web. Springer Science \& Business Media. 